\title{
Valorisation par voie hydrométallurgique de Matières Premières Secondaires
}

\section{Valorization of Secondary Raw Materials by hydrometallurgical way}

\author{
Eric Meux ${ }^{1}$, N. Leclerc ${ }^{1}$, C. Hazotte ${ }^{1}$, M. Schneider ${ }^{2}$ et F. Lapicque ${ }^{3}$ \\ 1 Institut Jean Lamour, Université de Lorraine, Metz, France \\ 2 LCP-A2MC, Université de Lorraine, Metz, France \\ 3 Laboratoire Réactions et Génie des Procédés, Université de Lorraine, ENSIC, Nancy, France
}

\begin{abstract}
This talk will focus on the hydrometallurgical recovery of metals contained in secondary raw materials. At first, generalities about critical metals, their recycling rate and their identified supply will be addressed. Two examples of hydrometallurgical treatment will be then developed: the treatment of spent hydrotreating catalysts and the treatment of electrodes powders coming from grinding of spent batteries by coupling electroleaching/electrodeposition.
\end{abstract}

Résumé. Cet exposé s'intéressera à la récupération par voie hydrométallurgique de métaux contenus dans les Matières Premières Secondaires. Dans un premier temps seront abordées des généralités sur les Métaux Critiques, leur taux de recyclage et les gisements identifiés. Deux exemples de traitement seront ensuite développés : le traitement de catalyseurs d'hydrotraitement et le traitement de poudres métallifères issues de broyats d'accumulateurs par couplage électrolixiviation/électrodéposition.

\section{INTRODUCTION}

De nombreux coproduits de la métallurgie (poussières, boues, scories), matériaux en fin de vie (catalyseurs de l'industrie chimie et pétrochimique) ou encore biens de consommation usagés (piles, batteries, Produits Electriques et Electroniques en Fin de Vie) contiennent des métaux et sont qualifiés à ce titre de Matières Premières Secondaires (MPS). Certains de ces déchets contiennent en effet des concentrations en métaux égales voire supérieures à celles que l'on trouve dans les minerais classiquement utilisés. Parmi ces métaux, on trouve la famille des Métaux Mineurs dont la production varie de quelques centaines de tonnes jusqu'à un maximum d'environ 150000 tonnes. C'est au sein de cette famille que l'Union Européenne a établi en juin 2010 une liste de métaux dits critiques, la notion de criticité étant basée sur la stabilité politico-économique des pays producteurs, le niveau de concentration de la production ainsi que les possibilités de substitution et le taux de recyclage (Fig. 1).

Si pour certains métaux le taux de recyclage atteint des valeurs très élevées, pour d'autres, tout reste à faire comme l'indique la figure 2 .

Le tableau 1 présente quelques exemples de matériaux et biens de consommations en fin de vie avec les métaux qu'ils contiennent.

Le traitement hydrométallurgique des Matières Premières Secondaires est schématisé sur la Figure 3.

Comme pour les minerais métalliques, la plupart des MPS doivent subir des traitements physiques avant d'envisager l'extraction sélective ou non des métaux par lixiviation chimique. Les coproduits de la métallurgie ainsi que les catalyseurs n'ont pas besoin de ces prétraitements.

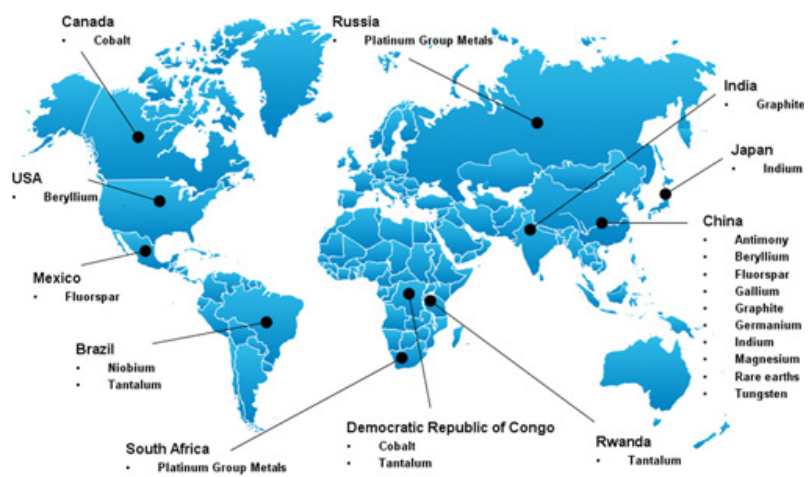

Figure 1. Les 14 matières premières minérales jugées critiques par la Commission Européenne [EC, 2010].

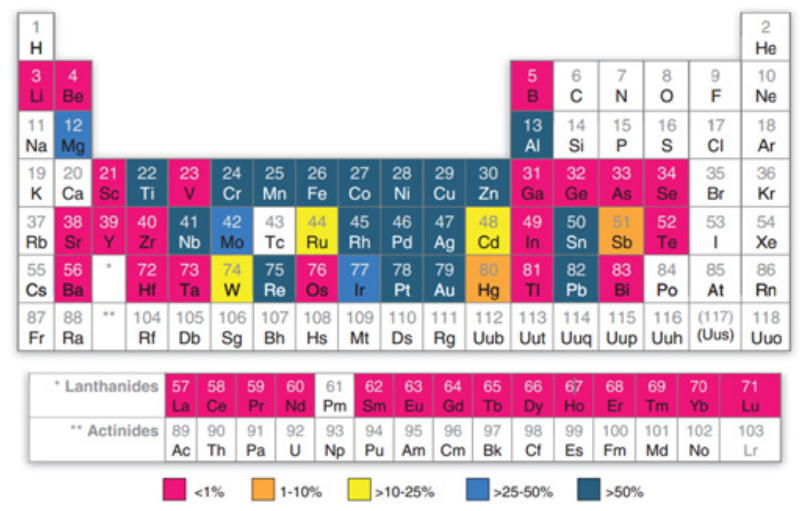

Figure 2. Estimations du taux de recyclage pour 60 métaux et non-métaux [Reck and Graedel, 2012].

Une des thématiques de recherche de l'équipe concerne la mise au point de protocoles chimiques et/ou électrochimiques permettant la récupération des métaux à partir de différents matrices par voie hydrométallurgique. 
Tableau 1. Exemples de matières premières secondaires contenant des métaux critiques.

\begin{tabular}{|l|c|}
\hline Matière Première Secondaire & Métaux récupérables \\
\hline Batteries Li-Ion & $\mathrm{Co}$ \\
\hline Batteries NiMH & $\mathrm{Ce}, \mathrm{La}, \mathrm{Nd}, \mathrm{Pr}, \mathrm{Sm}$ \\
\hline Catalyseurs & $\mathrm{Ce}, \mathrm{Co}, \mathrm{La}, \mathrm{Nd}, \mathrm{Pr}, \mathrm{W}$ \\
\hline Circuits imprimés & $\mathrm{Ta}$ \\
\hline Ecrans LCD & $\mathrm{In}$ \\
\hline Ecrans et ampoules LED & $\mathrm{Ga}$ \\
\hline Cellules photovoltaïques & $\mathrm{Ga}, \mathrm{In}$ \\
\hline $\begin{array}{l}\text { Tubes luminescents et ampoules } \\
\text { basse consommation }\end{array}$ & $\mathrm{Eu}, \mathrm{Tb}, \mathrm{Y}$ \\
\hline
\end{tabular}

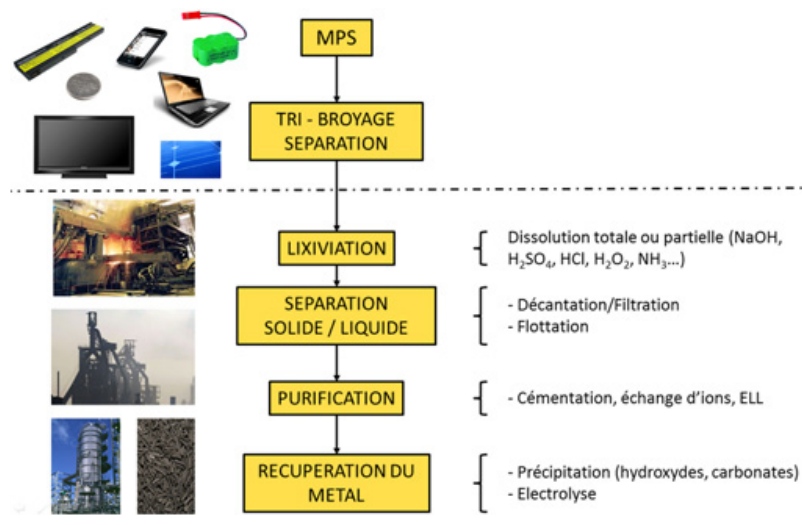

Figure 3. Traitement hydrométallurgique de MPS.

Deux exemples seront abordés au cours de cet exposé :

- La valorisation des catalyseurs d'hydrotraitement utilisés pour le raffinage des hydrocarbures

- La valorisation d'accumulateurs en fin de vie par électrolixiviation/électrodéposition.

\section{EXEMPLES DE VALORISATION}

\section{Catalyseurs usés d'Hydrotraitement}

Les procédés d'hydrotraitement des coupes pétrolières permettent d'éliminer les principaux polluants présents dans les pétroles bruts par des réactions d'hydrodémétallisation (HDM), d'hydrodésulfuration (HDS) ou encore d'hydrodénitruration (HDN). Ces opérations sont réalisées à l'aide de catalyseurs $\mathrm{CoMo} / \mathrm{Al}_{2} \mathrm{O}_{3}, \mathrm{NiW} / \mathrm{Al}_{2} \mathrm{O}_{3}$ ou encore $\mathrm{NiMo} / \mathrm{Al}_{2} \mathrm{O}_{3}$. La production de carburants dits 《 propres » à partir de pétroles de plus en plus lourds et l'accroissement de la demande conduisent à l'augmentation de la quantité de catalyseurs usés générée qui est comprise entre 150000 et 170000 tonnes par an [Dufresne, 2007].

La phase active des catalyseurs d'hydrotraitement est généralement constituée de sulfures métalliques dispersés sur une matrice d'alumine poreuse. Lors de leur utilisation, les catalyseurs sont soumis à divers phénomènes provoquant leur désactivation, dont notamment le dépôt de coke ou de métaux (essentiellement nickel et vanadium contenus dans les bruts pétroliers).

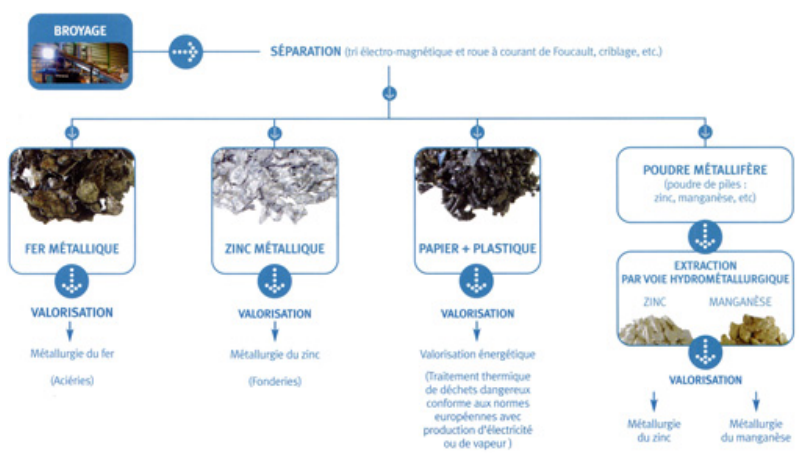

Figure 4. Traitement des piles $\mathrm{Zn} / \mathrm{MnO}_{2}$ mis en place chez EuroDieuze Industrie(57).

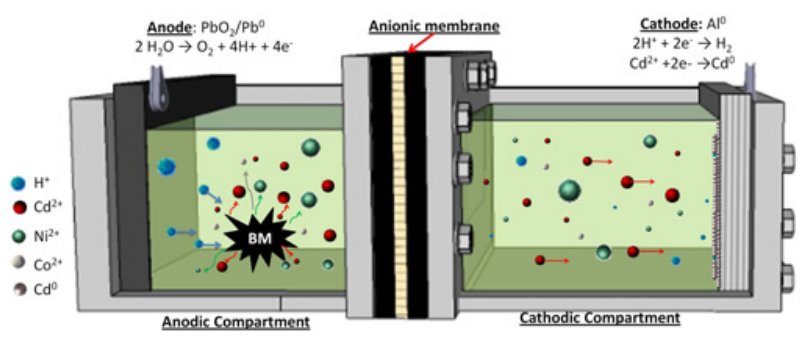

Figure 5. Couplage Electrolixiviation/Electrodéposition.

Voie classique : la première étape consiste à faire subir au catalyseur un grillage flash à environ $800^{\circ} \mathrm{C}$ qui a pour but de transformer les phases sulfurés en oxydes. Ensuite, les catalyseurs sont lixiviés par $\mathrm{NaOH}$ afin de solubiliser $\mathrm{MoO}_{3}$ et $\mathrm{WO}_{3}$ sous la forme de $\mathrm{MoO}_{4}^{2-}$ et $\mathrm{WO}_{4}^{2-}$. Ces ions sont ensuite précipités sous forme de sels calciques $\mathrm{CaMoO}_{4}$ et $\mathrm{CaWO}_{4}$ utilisables pour l'élaboration de ferromolybdène et de tungstène. Le nickel ou le cobalt présents dans le résidu de lixiviation sodique sont récupérés par voie pyrométallurgique [Ruiz et al., 2011].

Voie alternative : nous avons également étudié la possibilité de s'affranchir de l'étape de grillage en oxydant les sulfures métalliques soit par $\mathrm{H}_{2} \mathrm{O}_{2}$ en milieu acide, soit par $\mathrm{NaClO}$ en milieu basique. Dans le premier cas, on lixivie simultanément le molybdène et le cobalt avec des rendements de près de $90 \%$ sans attaquer de manière significative $\mathrm{Al}_{2} \mathrm{O}_{3}$. Dans le second cas, la lixiviation est sélective de Mo, extrait avec un rendement de $100 \%$.

\section{Accumulateurs usagés}

Les piles et accumulateurs collectés sont triés manuellement par famille. Après broyage, les différents constituants sont séparés (tri magnétique, tri à courant de Foucault). La poudre métallifère constituée des matériaux d'électrodes, communément appelée « Black Mass », est lixiviée par $\mathrm{H}_{2} \mathrm{SO}_{4}$ concentré. Les métaux solubilisés sont généralement récupérés par précipitation de carbonates et/ou d'hydroxydes. La Figure 4 présente l'exemple du traitement des piles $\mathrm{Zn} / \mathrm{MnO}_{2}$.

Nous étudions actuellement la possibilité de coupler les étapes de lixiviation et d'électrolyse dans une même cellule. Le principe (Fig. 5) est de générer à l'anode des protons par oxydation de l'eau qui vont provoquer la 
lixiviation des métaux et des oxydes métalliques contenus dans la Black Mass. Les cations migrent vers la cathode en traversant une toile polymère (ou une membrane anionique) où ils sont réduits. Des résultats obtenus avec des matériaux d'électrodes d'accumulateurs $\mathrm{NiCd}$ démontés manuellement seront présentés.

\section{Références}

EC (European Commission), Critical raw materials for the EU - Report of the Ad-hoc Working Group on defining critical raw materials, 30 july 2010.

B.K Reck and T.E Graedel, Challenges in Metal Recycling Science, 2012, 337 (6095), 690-695.
P. Dufresne, Hydroprocessing catalysts regeneration and recycling, Applied catalysis A-General, 2007, 322, $67-75$.

V. Ruiz, E. Meux, S. Diliberto and M. Schneider, Hydrometallurgical Treatment for Valuable Metals Recovery from Spent $\mathrm{CoMo} / \mathrm{Al}_{2} \mathrm{O}_{3}$ Catalyst. 1. Improvement of Soda Leaching of an Industrially Roasted Catalyst, Industrial and Engineering Chemistry Research, 2011, 50 (9), 5295-5306.

V. Ruiz, E. Meux, M. Schneider and V. Georgeaud, Hydrometallurgical Treatment for Valuable Metals Recovery from Spent $\mathrm{CoMo} / \mathrm{Al}_{2} \mathrm{O}_{3}$ Catalyst. 2. Oxidative Leaching of an Unroasted Catalyst Using $\mathrm{H}_{2} \mathrm{O}_{2}$, Industrial and Engineering Chemistry Research, 2011, 50 (9), 5307-5315. 